\section{Avaliação da implantação de programa de atenção pré-natal no Município de Curitiba, Paraná, Brasil: estudo em coorte de primigestas}

\author{
Evaluation of the prenatal care program \\ in Curitiba, Paraná, Brazil: \\ a cohort study of primigravidae
}

\author{
1 Departamento de Saúde \\ Comunitária, Universidade \\ Federal do Paraná, \\ Curitiba, Brasil. \\ 2 Faculdade de Medicina, \\ Universidade de São Paulo, \\ São Paulo, Brasil. \\ Correspondência \\ D. S. Carvalho \\ Departamento de Saúde \\ Comunitária, Universidade \\ Federal do Paraná. \\ Rua Padre Camargo 280, \\ Curitiba, $P R$ \\ 80060-240, Brasil. \\ denisecarvalho@ufpr.br
}

\begin{abstract}
A prenatal care program was evaluated to measure its effectiveness and impact. In 1999 a new prenatal care program was introduced in $\mathrm{Cu}$ ritiba, Paraná State, Brazil, in health services under the Unified National Health System, introducing organizational changes in order to enhance accessibility, increase the number and distribution of prenatal visits, and ensure minimum necessary procedures. To evaluate how these changes were assimilated in routine practice, a cohort study was proposed in a sample of 660 primigravidae randomly selected among women enrolled in the program before the 20th week of gestation, from March to August, 2000. Two home visits and consultation of maternity cards or patient records during prenatal and hospital care were performed. The results indicate good accessibility and professional adherence to the protocol, but problems still remain with initiating care before the end of the first trimester and adequate distribution of consultations. According to the study criteria, adequate care was found for only $38.6 \%$ of the women, pointing to the complexity of factors that influence adequate prenatal care.
\end{abstract}

Prenatal Care; Health Services; Services Evaluation
Denise Siqueira de Carvalho 1

Hillegonda Maria Dutilh Novaes 2

\section{Introdução}

A importância da avaliação em sistemas e serviços de saúde, para o contexto brasileiro, vem sendo ressaltada em diversos momentos da discussão das políticas de saúde e práticas em serviços, evidenciada pelo crescente volume de publicações sobre o assunto e presença nos encontros científicos de saúde pública, e objetivada ao se traduzir em movimentos institucionais dirigidos a inserir a prática da avaliação no planejamento e implementação de programas e ações na área da saúde. Essa tendência se insere em um contexto mundial, e em alguns países a avaliação de sistemas e serviços de saúde já se constitui em uma prática institucionalizada e seus resultados contribuem para as decisões nas suas políticas e práticas de saúde 1,2 .

No Brasil, a prática da avaliação, mais desenvolvida no que diz respeito ao uso de indicadores gerais de monitoramento no SUS, é ainda incipiente nos serviços de saúde. As avaliações acontecem, mais freqüentemente, em situações pontuais quando a necessidade e a oportunidade de se compreender o que ocorre no exercício do cotidiano da atenção à saúde, permitem a realização de pesquisas, habitualmente com participação da academia, que têm por objetivo estudar o desempenho dos serviços e produzir recomendações que orientem soluções para os problemas identificados. No 
entanto, a implementação de mudanças nas práticas se mostra dependente de condições que extrapolam a realização das pesquisas havendo um distanciamento e descontinuidade entre o momento da produção do conhecimento e as condições para a sua utilização no redirecionamento das práticas 3 .

Entre os programas ou ações programáticas em saúde, a assistência pré-natal tem ocupado historicamente um espaço relevante na atenção à saúde da população 4 , sendo um dos primeiros a ser desenvolvido com uma perspectiva de saúde pública 5 . Os programas de atenção pré-natal nos países ocidentais são ainda baseados no modelo de atendimento proposto por Janet Campbell, na Inglaterra no início do século $\mathrm{XX} 6$ e a ele foram acrescentados novos procedimentos, decorrentes do desenvolvimento científico e tecnológico, e introduzidas mudanças nas práticas, sem estarem necessariamente apoiados, tal qual na prática médica em geral, em avaliações quanto à sua efetividade, custos e justificativa clínica ou epidemiológica 7 .

Tendo por objetivo central reduzir a morbidade e mortalidade, tanto materna 8 quanto infantil 9 , o programa de pré-natal caracteriza-se pelo desenvolvimento de ações preventivas e educativas e, por meio do contato freqüente e planejado da gestante com os serviços de saúde, no rastreamento de gestantes de alto risco e intervenção precoce nos problemas que afetam a gravidez.

No Brasil, a disseminação, de forma mais ampla, do programa de atenção pré-natal ocorreu com a implantação do Programa de Atenção Integral à Saúde da Mulher (PAISM), em meados da última década de 80 . Desde esse período, têm ocorrido mudanças significativas quanto ao aumento da cobertura e da média do número de consultas, bem como quanto ao início mais precoce do pré-natal. Os indicadores de saúde relativos à morbidade e à mortalidade materna e perinatal, embora venham apresentando melhoras, continuam insatisfatórios em todas as regiões do país, ainda que em níveis diversos, e considera-se que poderiam ser bastante melhorados com o aprimoramento dos programas implantados 10,11.

Ainda que inquestionável o potencial para um impacto positivo do pré-natal sobre a saúde da população, enquanto ação tecnicamente orientada e voltada para uma condição essencial para a saúde em geral, têm sido propostos, nos últimos anos, análises que buscam compreender melhor as articulações entre os seus resultados com as condições socioeconômicas e de vida de grupos sociais específicos. Essas condições, para além dos efeitos próprios sobre a saúde, condicionam também os resultados da atenção à saúde, pela acessibilidade sempre mais difícil, por razões as mais diversas, mesmo quando os recursos existem, e pela dificuldade em implementar as recomendações que propiciariam um "comportamento saudável" e uma detecção mais precoce de problemas potencialmente de risco 12,13 .

Uma outra vertente de pesquisas busca identificar quais são as intervenções, quanto ao tipo e intensidade, que apresentam melhor associação com resultados positivos sobre os indicadores de morbidade e mortalidade, com ênfase no momento do início do acompanhamento, número de consultas, o papel das atividades educativas e procedimentos de rastreamento, diagnósticos e terapêuticos essenciais 14,15. A falta de consenso quanto aos critérios que definem um pré-natal adequado e a dificuldade em controlar nos resultados a participação dos contextos socioeconômicos e culturais populacionais, das características dos sistemas e serviços de saúde e das relações entre os profissionais e sua clientela, fazem com que os resultados das avaliações se mostrem difíceis de serem comparados e generalizados 16,17,18,19.

O programa de assistência pré-natal do Município de Curitiba, Paraná, Brasil, desde a sua implantação em 1979, passou por mudanças que acompanharam a evolução histórica dos serviços de saúde, como a ampliação da cobertura da atenção médica, a hierarquização dos níveis de atenção, a participação comunitária, a municipalização e a distritalização. O conteúdo do programa, formulado com base nas diretrizes do PAISM e das normas do Ministério da Saúde e adaptado à realidade das unidades de saúde, permaneceu inalterado até 1999, quando foi elaborada uma nova proposta, após revisão dos objetivos e correção de deficiências apontadas em avaliações anteriores 20 , sendo implantado um novo programa que passou a ser conhecido como "Programa Mãe Curitibana". Nesse novo modelo reorganizou-se o fluxo de atendimento da população usuária do SUS, redefiniu-se a quantidade e a distribuição das consultas, e estabeleceram-se normas bem definidas acerca dos procedimentos necessários a um pré-natal de qualidade, que foram adotados pelas 92 unidades básicas de saúde do município que prestam atendimento programado à população de sua área de abrangência e que 
corresponde a aproximadamente $65,0 \%$ do total de gestantes do município.

Essas mudanças, específicas para a saúde materno-infantil no contexto da atenção básica, foram complementadas por investimentos na atenção hospitalar, organizando o fluxo da clientela a ser atendida de acordo com o grau de complexidade da atenção necessária e vinculação da gestante à maternidade. Ações com vistas à maior humanização na atenção ao parto foram pactuadas com os serviços hospitalares, introduzindo-se visitas das gestantes aos hospitais de vinculação, garantia de acesso e melhorias na qualidade dos procedimentos realizados durante o internamento.

O protocolo adotado e a implantação de fluxos sistematizados resultaram do trabalho conjunto desenvolvido pelos serviços de saúde vinculados ao SUS e por sociedades científicas, tendo recebido o aval do Conselho Municipal de Saúde de Curitiba 21 . As diferenças em relação ao modelo de atenção anterior, referentes à introdução de alguns procedimentos e o reforço de outros já previstos, mas realizados de forma não sistemática, compreenderam:

(a) Definição das unidades de saúde como porta de entrada para a assistência pré-natal de todas as gestantes usuárias do SUS, vinculandoas desde o início a uma maternidade de referência;

(b) Realização de procedimentos que antecederiam, agilizariam e otimizariam a primeira consulta médica no momento da inscrição da gestante ao programa, como a abertura de prontuário, a entrega da carteira da gestante e de materiais educativos, e a realização dos exames de rotina: eritrograma, tipagem sangüínea, VDRL, sorologia para toxoplasmose e parcial de urina;

(c) Introdução na rotina do pré-natal do teste de tolerância à glicose, dispensando a glicemia de jejum no rastreamento do diabetes gestacional; (d) Introdução do uso da imunoglobulina antiRh na 28a semana de gestação para as mulheres com Rh negativo e com resultado negativo para o teste de Coombs indireto, este realizado até a 24a semana;

(e) Garantia de sete consultas médicas, distribuídas ao longo do pré-natal, sendo uma no primeiro, duas no segundo e quatro no terceiro trimestre, intercalando consultas de enfermagem; (f) Análise do risco gestacional em todas as consultas médicas e realização do acompanhamento de gestantes de baixo risco e de risco controlado na própria unidade de saúde, encaminhando-as à assistência de alto risco, quando houver necessidade de atendimento médico-hospitalar de maior complexidade; (g) Monitoramento do comparecimento da gestante ao programa, com realização de visitas domiciliares para busca ativa das gestantes faltosas;

(h) Exigência do preenchimento do cartão da gestante e de prontuários com dados referentes a todos os procedimentos realizados e à avaliação do risco gestacional.

Um ano após a introdução na dinâmica dos serviços dos princípios e normas definidos pelo programa, foi possível considerar estar concluído o seu processo de implantação gerencial e organizacional. Colocou-se, então, a necessidade de avaliar de forma sistemática, os processos de atenção pré-natal na prática cotidiana dos profissionais, tomando por referência as diretrizes técnicas estabelecidas pelo programa. De início, mostrou-se necessário o dimensionamento da efetiva implantação do programa para que fosse possível uma posterior avaliação mais fundamentada dos resultados e impacto do mesmo.

O projeto, cujos resultados iniciais são aqui apresentados, estudou de forma prospectiva, condição rara nas pesquisas brasileiras sobre o tema, uma coorte de primigestas com inscrição precoce no programa, buscando garantir uma compreensão mais precisa da dinâmica dos processos assistenciais gerais e o fluxo dos processos individuais. Foram utilizadas diversas fontes de informações, com coletas de dados primários em entrevistas domiciliares e dados secundários nos documentos oficiais (carteira da gestante e prontuários). A escolha por primigestas teve o propósito de minimizar a influência de experiências de gestações anteriores nos conhecimentos e práticas relacionados à gravidez e ao parto, bem como na forma de utilização dos serviços durante o pré-natal. Também a inscrição precoce, como critério de inclusão, teve a intenção de selecionar aquelas mulheres que pudessem usufruir de todos os benefícios previstos no programa, permitindo uma maximização dos resultados do programa.

\section{Metodologia}

O estudo envolveu uma coorte de primigestas usuárias do SUS, inscritas no Programa Mãe Curitibana, com idade gestacional máxima de vinte semanas, e com data provável de parto entre agosto de 2000 e janeiro de 2001. O cálculo do tamanho da amostra foi realizado estabelecendo-se como erros estatísticos máximos de $5,0 \%$ para o erro de tipo I e $20,0 \%$ para o erro de tipo II, e um poder de $80,0 \%$ para o estudo. Para um desvio de $3,0 \%$, para mais ou para 
menos, nas estimativas efetuadas e estimando a população de referência em aproximadamente 2.700 primigestas atendidas pelo SUS e que ingressaram precocemente no programa no período do estudo, foi definida uma amostra de 660 mulheres, com desvio aproximado de $2,5 \%$.

Foram selecionadas aleatoriamente 110 mulheres/mês, no período de março a agosto de 2000, totalizando uma amostra inicial de 660 primigestas. Duas visitas domiciliares, a primeira antes da 28a semana de gestação e a segunda aproximadamente trinta dias após o parto foram realizadas, com coleta de informações quanto ao perfil sócio-econômico, condições gerais de saúde e sobre a atenção realizada no pré-natal, após consulta à carteira da gestante ou ao prontuário ambulatorial, e sobre o parto, após consulta ao prontuário hospitalar e à declaração de nascidos vivos.

Para a definição da adequação do pré-natal, utilizaram-se indicadores elaborados a partir das metas estabelecidas pelo programa. Foram definidos três indicadores básicos: adequação do número e distribuição das consultas médicas; procedimentos essenciais e adequação geral, compreendendo a somatória dos anteriores. Como critério do primeiro indicador, definiu-se como adequado um mínimo de sete consultas distribuídas da seguinte forma: uma consulta no primeiro trimestre, duas no segundo trimestre e quatro no terceiro. Para o indicador de procedimentos mínimos, considerouse que a proporção de registros de pressão arterial, de medidas do peso materno, da altura uterina e dos batimentos cardíacos fetais, ponderados pelo número de consultas realizadas, seriam adequados se estivessem presentes em no mínimo 70,0\% das consultas, associados à presença do registro de pelo menos quatro dos procedimentos previstos: exame laboratorial de tipagem sangüínea, exame de VDRL, dosagem de hemoglobina, teste simplificado de tolerância à glicose ou glicemia de jejum, avaliação da apresentação fetal e vacinação antitetânica. O indicador de adequação geral foi definido pela reunião dos dois indicadores. Esses parâmetros foram definidos baseados em propostas referidas na literatura e de recomendações de associações médicas 14,22,23,24.

Os dados coletados em formulários específicos foram codificados e digitados em quatro bancos de dados separados, validados quanto à sua consistência e submetidos à análise estatística utilizando-se o programa Epi-Info versão 6.04b. Os dados aqui apresentados têm por objetivo caracterizar a implantação e dinâmica de desempenho do programa nos serviços de saúde e o grau de adequação encontrado na coorte. A análise dos fatores que se associaram com a adequação encontrada e dos resultados e impacto do programa serão apresentados em outra publicação.

\section{Resultados}

No período de seleção da amostra, 6.434 gestantes inscreveram-se no programa com idade gestacional inferior a vinte semanas. Dentre 5.628 que dispunham da informação do número de gestações anteriores, foi possível identificar 2.207 (39,2\%) primigestas, das quais foram selecionadas as 660 mulheres que compuseram a coorte a ser estudada.

Desse contingente inicial, 198 mulheres (30,0\%) não puderam ser incluídas no estudo. Dificuldade na localização de 104 mulheres devido à inexistência do endereço fornecido pelas unidades de saúde, mudança para endereço ignorado ou para outros municípios, foram os motivos responsáveis pela não inclusão de $15,8 \%$ do total. A inelegibilidade de $72(10,9 \%)$ mulheres por erro de classificação (não ser primigesta), não estar grávida no momento da primeira visita domiciliar devido a aborto, ou pelo fato de a criança já ter nascido, não ter sido confirmada a gravidez, mudança para serviço de assistência pré-natal não vinculado ao SUS e erros no processo de cadastramento da usuária no sistema também contribuiu para essa redução no número de casos. A recusa de participação na pesquisa representou a perda de 22 mulheres $(3,3 \%)$ do total inicialmente selecionado.

Passaram a constituir o grupo de coorte, 462 primigestas usuárias do SUS, residentes em Curitiba, inscritas no programa até a vigésima semana de gravidez e com data provável de parto no período de agosto de 2000 a janeiro de 2001. Na segunda visita, vinte mulheres não puderam ser entrevistadas por motivo de mudança para outro município ou para endereço ignorado e quatro recusas. Dentre as $442 \mathrm{mu}$ lheres localizadas, 408 permaneceram vinculadas ao programa até o final da gravidez, das quais, obtiveram-se os dados ambulatoriais e hospitalares de 376 (92,2\%). As que deixaram o programa, 34 mulheres, o fizeram por preferirem outros serviços (Figura 1).

Quanto às características sócio-demográficas, a média de idade das mulheres da coorte foi de 20,4 anos, 51,3\% com menos de vinte anos, sendo a maioria casada $(75,8 \%)$, com nível de escolaridade equivalente ao primeiro grau completo ou maior em $60,0 \%$ dos casos e renda per capita média de $\mathrm{R} \$ 205,12$. 
Figura 1

Fluxograma de identificação da amostra e da coleta de dados.

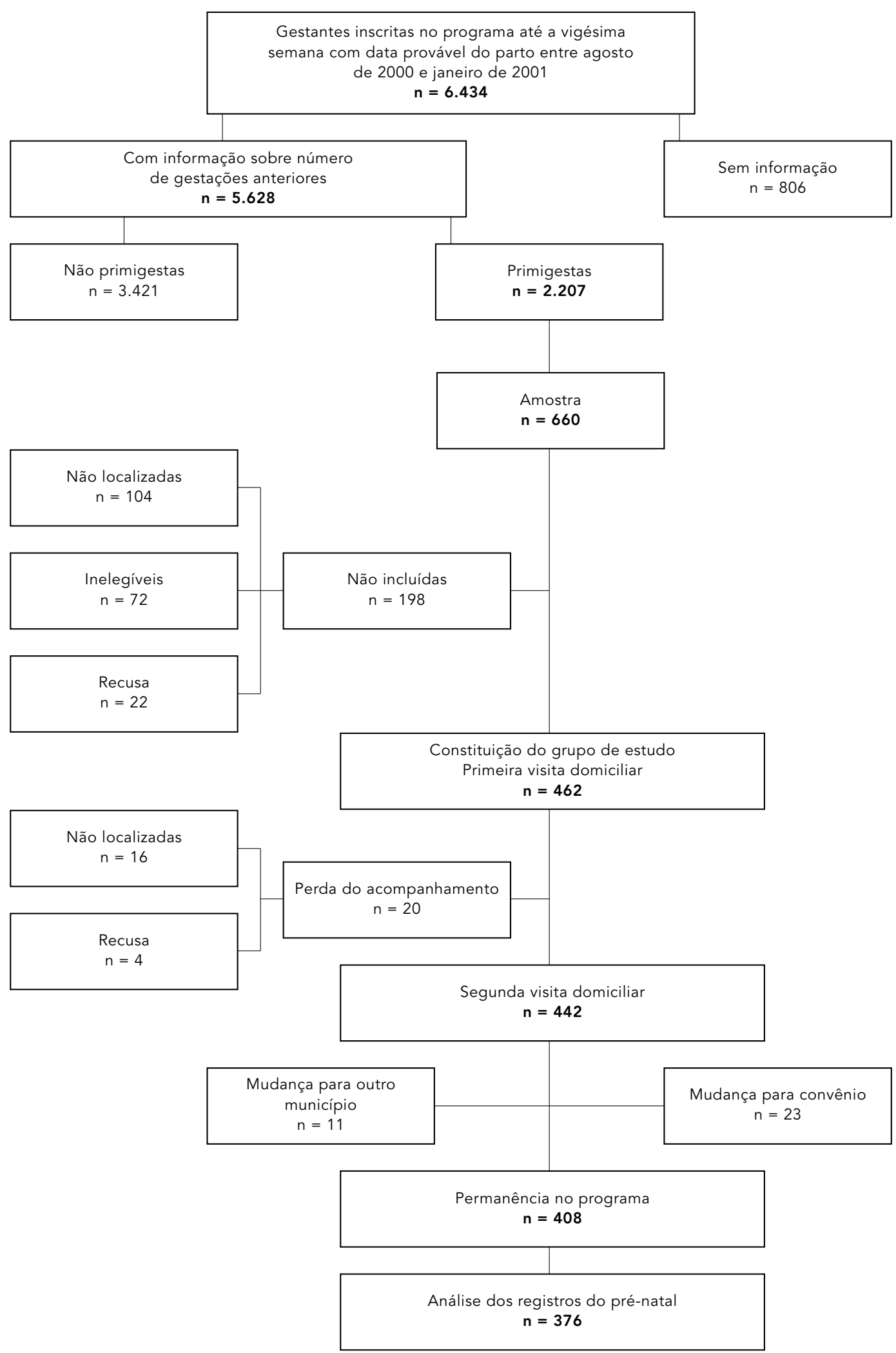


No que diz respeito à oferta de serviços e acessibilidade, a proximidade da unidade de saúde em relação à residência da entrevistada foi o motivo da escolha do local para a realização do acompanhamento pré-natal (84,8\%), e o tempo médio de deslocamento até a unidade de saúde era de 14 minutos. A busca do serviço de saúde após a constatação da gravidez apresentou um intervalo de 28 dias em média e 69 mulheres $(14,9 \%)$ relataram dificuldades para o atendimento inicial e/ou para a inscrição no programa, sendo a demora no atendimento, problemas no cadastramento no programa e disponibilidade de consultas médicas as principais queixas. No decorrer da gestação, o agendamento das consultas médicas foi apresentado como uma dificuldade para 21 mulheres $(5,2 \%)$ vinculadas ao programa e entre as que dele se desvincularam, essa dificuldade foi mencionada por $18,2 \%$. Das 408 mulheres que permaneceram vinculadas ao programa, 350 $(85,8 \%)$ realizaram seu pré-natal sempre no mesmo local e 279 (79,6\%) foram atendidas sempre pelo mesmo profissional.

A média do número de atendimentos realizados na unidade de saúde, de acordo com as entrevistadas, foi de 10,7 e a média do número de consultas médicas foi de 8,9. O número de consultas médicas, obtido por meio da contagem das datas de consulta registradas na carteira da gestante ou prontuário ambulatorial foi em média de 8,6 e observou-se um número mínimo de 3 e máximo de 16 consultas (Tabela 1).

Com relação à distribuição das consultas de acordo com o trimestre de gravidez, observou-se nos registros que para $132(35,1 \%)$ não havia registro de consulta médica no primeiro trimestre de gravidez, considerado como tal o período até a 13a semana, inclusive. Durante o segundo trimestre, a média de consultas registradas foi de 3,2 e $364(96,8 \%)$ realizaram duas ou mais consultas no período. No terceiro trimestre, a média de consultas foi de 4,3 com um mínimo de 0 e um máximo de 11 consultas no período, sendo que $220(58,5 \%)$ realizaram quatro ou mais consultas no trimestre. Duas das mulheres que tiveram parto pré-termo, foram consideradas como adequadas, pois realizaram o número recomendado de consultas para a sua idade gestacional.

$\mathrm{Na}$ avaliação dos procedimentos que fazem parte da rotina do primeiro atendimento, conforme informações obtidas na entrevista, todos os procedimentos foram realizados na sua quase totalidade, exceto a coleta de exame para confirmação da gravidez e a consulta médica no mesmo dia (Tabela 2).

Os procedimentos de rotina adotados durante as consultas de pré-natal de medida da
Tabela 1

Número de consultas realizadas no pré-natal conforme dados da entrevista e consulta aos registros.

\begin{tabular}{|c|c|c|c|c|}
\hline \multirow[t]{2}{*}{ Número de consultas } & \multicolumn{2}{|c|}{ Entrevista $(n=408)$} & \multicolumn{2}{|c|}{ Registros $(n=376$ ) } \\
\hline & $\mathrm{n}$ & $\%$ & $n$ & $\%$ \\
\hline Até 4 & 25 & 7,3 & 13 & 3,5 \\
\hline 5 a 6 & 45 & 14,0 & 63 & 16,8 \\
\hline 7 a 9 & 147 & 41,6 & 169 & 44,9 \\
\hline 10 e mais & 131 & 37,0 & 131 & 34,8 \\
\hline Total & 348 & 100,0 & 376 & 100,0 \\
\hline Sem informação & 60 & 14,3 & - & - \\
\hline
\end{tabular}

Tabela 2

Procedimentos de rotina do primeiro atendimento pré-natal

de acordo com a primeira entrevista domiciliar.

\begin{tabular}{|c|c|c|}
\hline \multirow[t]{2}{*}{ Procedimentos } & \multicolumn{2}{|c|}{ Realizados $(n=462)$} \\
\hline & $\mathrm{n}$ & $\%$ \\
\hline Exame confirmatório da gravidez & 330 & 71,4 \\
\hline Recebimento da pasta da gestante & 435 & 94,1 \\
\hline Recebimento da carteira da gestante & 442 & 95,7 \\
\hline Medida da pressão arterial & 452 & 97,8 \\
\hline Verificação do peso & 450 & 97,4 \\
\hline Verificação da estatura & 436 & 94,4 \\
\hline Consulta médica no mesmo dia & 358 & 77,5 \\
\hline Solicitação de exames de sangue & 441 & 95,5 \\
\hline Solicitação de exames de urina & 438 & 94,8 \\
\hline Orientações sobre o pré-natal & 410 & 88,7 \\
\hline Informação sobre o hospital do parto & 441 & 95,5 \\
\hline
\end{tabular}

pressão arterial, medida do peso e a ausculta dos batimentos cardíacos fetais foram referidos como sempre realizados por $98,8 \%, 97,3 \%$ e $97,3 \%$ das entrevistadas, respectivamente. A ausculta cardíaca e pulmonar da própria gestante foi referida por $61,5 \%$ delas como nunca realizada, e 1,0\% afirmou nunca ter sido medida a altura uterina (Tabela 3 ).

Sobre as orientações recebidas durante o pré-natal relacionadas à alimentação adequada, ao uso de preservativo, às atividades físicas, à prevenção de doenças sexualmente transmissíveis e à prevenção ao consumo de cigarro, álcool ou drogas (Tabela 4), 13,5\% informaram não terem recebido nenhuma das orientações mencionadas, enquanto $32,4 \%$ referiram terem-nas recebido integralmente, considerando-se as mulheres que permaneceram vincula- 
Tabela 3

Procedimentos realizados nas consultas de pré-natal conforme a percepção das entrevistadas que se mantiveram vinculadas ao programa.

\begin{tabular}{lccc}
\hline Procedimentos & \multicolumn{2}{c}{$\begin{array}{c}\text { Vinculadas ao programa } \\
\text { (n = 408) } \\
\text { Às vezes (\%) }\end{array}$} & Nunca (\%) \\
\hline Medida de peso materno & Sempre (\%) & Anco & - \\
Medida da pressão arterial & 97,3 & 2,7 & 0,2 \\
Medida da altura uterina & 98,8 & 1,0 & 1,0 \\
Ausculta cardíaca e pulmonar & 92,2 & 6,9 & 61,5 \\
Ausculta dos batimentos cardíacos fetais & 97,3 & 13,2 & 0,2 \\
Preenchimento do cartão da gestante & 95,3 & 4,4 & 0,2 \\
\hline
\end{tabular}

Tabela 4

Orientações fornecidas durante o pré-natal conforme a percepção das entrevistadas que se mantiveram vinculadas ao programa.

\begin{tabular}{|c|c|c|}
\hline \multirow[t]{2}{*}{ Orientações } & \multicolumn{2}{|c|}{ Entrevistadas $(n=408)$} \\
\hline & $\mathrm{n}$ & $\%$ \\
\hline Alimentação saudável & 291 & 71,3 \\
\hline Atividade física & 227 & 55,6 \\
\hline Prevenção ao consumo de cigarro, álcool e drogas & 288 & 70,6 \\
\hline Uso do preservativo & 199 & 48,8 \\
\hline Prevenção de doenças sexualmente transmissíveis & 239 & 58,6 \\
\hline Identificação de sinais de complicações & 260 & 63,7 \\
\hline Aleitamento materno & 347 & 85,0 \\
\hline Preparação para o parto & 219 & 53,7 \\
\hline Cuidado com o recém-nascido & 254 & 62,3 \\
\hline
\end{tabular}

das ao programa até o final da gravidez.

Quanto às orientações específicas sobre complicações da gravidez, preparação para o parto, aleitamento materno e cuidado com o recém-nascido $38,2 \%$ das mulheres informaram terem recebido todas as orientações, enquanto que $9,3 \%$ relataram não terem recebido orientação alguma. Em relação à necessidade de realização de consultas no pós-parto e para avaliação do desenvolvimento do recém-nascido, essas orientações foram recebidas por $77,9 \%$ das mulheres, porém $8,1 \%$ relataram não terem sido orientadas sobre isso.

A informação sobre o hospital para o qual deveriam dirigir-se no momento do parto foi recebida por $98,3 \%$ das mulheres vinculadas ao programa, sendo essa a mesma proporção das que levaram o cartão da gestante ao hospital.
Nos registros priorizou-se, na análise dos procedimentos realizados, a presença de informações referentes às medidas da pressão arterial (presente em 98,1\%), do peso materno $(91,9 \%)$, da altura uterina $(75,5 \%)$ e dos batimentos cardíacos fetais $(63,5 \%)$ registrados no mínimo em $70,0 \%$ das consultas realizadas, a estatura materna $(90,1 \%)$ e a avaliação da apresentação fetal $(72,9 \%)$, registradas pelo menos uma vez.

A aplicação da vacina antitetânica foi relatada por $91,0 \%$ das entrevistadas, sendo que $84,0 \%$ receberam duas doses ou mais. Nos registros, a informação da aplicação da vacina antitetânica, presente em 87,2\%, fazia menção à aplicação de duas ou mais doses em $81,4 \%$ desses.

Quanto à realização dos exames laboratoriais previstos na rotina do pré-natal, medida a partir da anotação dos seus resultados, verificou-se que os exames de triagem para sífilis e a tipagem sangüínea foram registrados em 375 casos $(99,7 \%)$, a dosagem de hemoglobina constava em $356(94,7 \%)$ e o teste simplificado de tolerância à glicose ou de glicemia de jejum foi registrado em $200(53,2 \%)$ dos 376 registros avaliados.

A categorização do pré-natal como adequado ou inadequado foi desenvolvida utilizandose as informações provenientes do cartão da gestante e dos prontuários ambulatoriais. $\mathrm{O}$ parâmetro de adequação de sete consultas durante o pré-natal foi observado em 302 dos 376 registros avaliados $(80,3 \%)$, incluídas as mulheres com parto pré-termo. A distribuição das consultas por trimestre de gestação, obedecendo-se ao previsto no programa, mínimo de uma no primeiro trimestre, duas no segundo e quatro no terceiro, apresentou-se satisfatório em 160 (42,6\%).

Quanto ao conjunto dos procedimentos, considerando-se aqueles registrados de forma seriada e os que são realizados pontualmente durante o pré-natal, observou-se que para 331 mulheres $(88,0 \%)$ os procedimentos foram considerados adequados (Tabela 5).

A reunião do critério de número de consultas e sua distribuição com o critério relativo aos procedimentos previstos para o pré-natal levaram ao cálculo de um critério de adequação geral. De acordo com tal critério, 145 das 376 mulheres avaliadas $(38,6 \%)$ tiveram um pré-natal adequado.

\section{Discussão}

Para a mensuração dos resultados de intervenções diagnósticas e terapêuticas, os estudos 
Critérios de adequação relativos aos procedimentos realizados durante o pré-natal.

\begin{tabular}{|c|c|c|c|c|}
\hline \multirow[t]{2}{*}{ Critério de adequação } & \multicolumn{2}{|c|}{ Adequado } & \multicolumn{2}{|c|}{ Inadequado } \\
\hline & $\mathrm{n}$ & $\%$ & $\mathrm{n}$ & $\%$ \\
\hline $\begin{array}{l}\text { Registro médio } \geq 70,0 \% \text { dos procedimentos realizados (medida de } \\
\text { peso, pressão arterial, altura uterina e ausculta de batimentos fetais) }\end{array}$ & 352 & 93,6 & 24 & 6,4 \\
\hline $\begin{array}{l}\text { Registro de outros procedimentos realizados } \\
\text { (exames laboratoriais, avaliação da situação fetal e vacina } \\
\text { antitetânica) mínimo de quatro, de um total de seis procedimentos }\end{array}$ & 351 & 93,4 & 25 & 6,6 \\
\hline Adequação do conjunto de procedimentos realizados & 331 & 88,0 & 45 & 12,0 \\
\hline
\end{tabular}

randomizados e controlados são considerados como a melhor opção metodológica ao buscar controlar outras condições que influenciam esses resultados, as variáveis de confusão 25 . Estudos dessa natureza, relacionados à assistência pré-natal, incluem desde a análise de procedimentos específicos 8,9,23 até aqueles que buscam abranger um conjunto de práticas, adequados à realidade social e econômica de cada localidade 26,27. Esses estudos, embora apresentem inúmeras contribuições, sofrem limitações ao incluir conjuntos articulados de intervenções e pela dificuldade em controlar a influência das condições reais em que se desenvolvem os programas 1 .

Estudos de coorte apresentam vantagens ao permitir a observação de fatores e condições do contexto em que se desenvolvem os programas, dificilmente captados em outros desenhos de pesquisa. O estudo realizado, de caráter prospectivo, voltado à verificação da implantação e impacto do protocolo de atenção pré-natal, e que tinha como pressuposto a existência de infra-estrutura material e organizacional necessária para o desenvolvimento do programa, evidenciou a interferência de fatores não previstos inicialmente, mas com reflexos importantes no desempenho do programa. De um lado, fatores ligados às questões administrativas, gerenciais e de controle do programa, como falhas no sistema de cadastramento e no processo de atualização e fluxos de informação, falta de recursos humanos qualificados; e de outro, fatores externos ao programa, como a grande mobilidade das mulheres nos espaços urbanos e a sua opção de busca por atendimento em locais que atendam às suas preferências independentemente do planejado pelos gestores do sistema, com conseqüências significativas na operacionalização do programa (Figura 1).

A coerência entre os dados primários e secundários obtidos na pesquisa ratificou a hipó- tese inicial de que a ausência de registros corresponderia à não realização dos procedimentos, critério também presente em outros estudos 28,29 (Tabela 1).

Poucos trabalhos abordam a primeira gravidez, observando-se na literatura a preferência por recortes relativos aos aspectos sóciodemográficos ou de condições de saúde. A opção pelo estudo das primigestas e com início do pré-natal até vinte semanas de gestação teve por objetivo identificar um grupo de gestantes potencialmente com maior valorização da atenção médica, ainda não influenciado por experiências anteriores, com menor prevalência de doenças crônicas, constituindo-se, portanto, em um grupo com condições mais favoráveis para um melhor desempenho do programa. No entanto, o elevado porcentual de gestantes adolescentes entre as primigestas, observado na pesquisa, introduziu questões específicas que relativizam essas características favoráveis quanto ao acompanhamento do prénatal, pois os estudos desenvolvidos sobre esse tema apontam o risco aumentado de complicações maternas e fetais, e as repercussões psicológicas e sociais da gestação nessas mulheres 30,31 . Associada a esses fatores, a insatisfação com a gravidez, significativamente maior no grupo de adolescentes do que entre as mulheres com vinte anos ou mais e observada também em outros trabalhos, pode conferir a esse grupo um comportamento desfavorável em relação à assistência à saúde e em particular com o pré-natal 32,33.

Quanto ao desempenho geral do programa, os resultados indicam uma acessibilidade satisfatória, observando-se pequena proporção de perda de acompanhamento e mudança para convênio ao longo da gestação e boa continuidade no atendimento. Isto é, das primigestas que iniciaram o pré-natal até a vigésima semana, puderam ser localizadas, eram elegíveis 
e aceitaram participar (462), a grande maioria permaneceu vinculada ao programa até o final da gestação, mas elas talvez configurem um grupo de mulheres com características particularmente mais favoráveis para esse comportamento.

Quanto ao início do acompanhamento, mesmo nesse grupo, apenas $65,0 \%$ das gestantes realizaram sua primeira consulta médica ainda no primeiro trimestre de gravidez, havendo referência a dificuldades persistentes na atenção precoce das gestantes e retardamento nas consultas e na realização dos exames de rotina. Quanto ao número médio de consultas, embora acima do mínimo exigido, mostrou espaçamento deficiente quando avaliado por meio dos parâmetros estabelecidos pelo programa, sendo o primeiro e terceiro trimestres os pontos críticos. Os valores encontrados mostram-se semelhantes a outros estudos brasileiros, mas devido às diferenças metodológicas, a comparabilidade fica prejudicada 11,34,35,36.

No primeiro trimestre, a realização da primeira consulta depende, em grande parte, da iniciativa da gestante, que está condicionada por um conjunto complexo de fatores, em que a disponibilidade e acessibilidade ao programa é parte importante mas não exclusiva. Uma vez procurado o serviço, algumas dificuldades administrativas se mantiveram na marcação da consulta, mas essa, quando realizada, cumpriu de forma satisfatória o protocolo proposto (Tabela 2).

No último trimestre, o número insuficiente de consultas teria como possíveis explicações, a persistência de barreiras relativas ao acesso e o não estabelecimento de vínculos com o serviço de pré-natal, associado à proximidade do período do parto, passando o hospital a ser a referência da gestante como local de busca, quando da ocorrência de sinais que possam sugerir o início do trabalho de parto, desvinculandose assim do atendimento da Unidade de Saúde.

Com relação aos procedimentos (Tabela 3), as medidas da pressão arterial e do peso materno foram os mais registrados durante o prénatal, o que reflete sua assimilação como prática rotineira das consultas médicas, mas a não realização freqüente da ausculta cardíaca e pulmonar pode indicar uma excessiva restrição na abordagem clínica. A medida da altura uterina e a ausculta dos batimentos cardíacos fetais, embora menos freqüentes, foram feitas com regularidade após o primeiro trimestre de gravidez, estando registradas de acordo com o recomendado. Isso significa que, tendo sido realizada a consulta médica, esses procedimentos também o foram, bem como os exames laboratoriais de tipagem sangüínea, dosagem de he- moglobina e triagem para sífilis. No entanto, a ausência de avaliação da apresentação fetal e o não registro do exame para triagem de diabetes, observados em $27,1 \%$ e $46,8 \%$ dos registros, respectivamente, é preocupante pela possibilidade de estar sendo perdida a oportunidade de diagnósticos precoces e prevenção de complicações. No caso dos exames para a diabetes, a média de idade das mulheres e história reprodutiva pouco sugestiva, aliada à introdução de um método de rastreamento da doença de maior complexidade, podem explicar a baixa aceitação por profissionais e usuárias. De uma maneira geral, os resultados quanto aos procedimentos nas consultas se mostram mais favoráveis do que os referidos na literatura, ainda que não totalmente satisfatórios 29 .

As orientações prestadas às gestantes constituem uma parte importante das ações realizadas durante o pré-natal, visando a preparação física e emocional da gestante, oferecendolhe subsídios para a compreensão do processo da gestação e o parto, bem como para os cuidados com o recém-nascido 23 . Embora relevantes, as orientações previstas na proposta do programa foram relatadas como integralmente abordadas apenas por $1 / 3$ das mulheres, e 1/10 afirmou não ter recebido nenhuma orientação. Embora se possa admitir que as entrevistadas não se lembrassem com precisão dos temas que foram abordados, o relato pode ser considerado indicativo do seu resultado (Tabela 4).

No contexto dos países desenvolvidos, as avaliações relativas ao pré-natal ocupam um grande espaço, mas têm sido ainda insuficientes na demonstração do pré-natal ideal e do seu impacto na saúde materna e infantil 18. Estudos adicionais são apontados como necessários, mesmo nesses países onde as taxas de mortalidade materna e infantil têm atingido níveis mínimos, para a identificação dos componentes do pré-natal capazes de reduzir os efeitos das desigualdades sociais e econômicas que afetam a saúde da mãe e do feto.

No Brasil, alguns estudos sobre o pré-natal têm se voltado para, além dos indicadores relativos ao início do tratamento, quantidade e distribuição de consultas, outras medidas que discriminem elementos do seu conteúdo 29,35,37,38. Denotam a preocupação de compreender de que forma a atenção pré-natal tem sido dispensada à população sem, no entanto, distinguir quais as mudanças das condições de saúde são decorrentes desse cuidado.

O objetivo geral da pesquisa foi de avaliar se a estratégia de cuidado selecionada e implantada pelo protocolo do pré-natal teve a capacidade de modificar as condições de saúde 
da mãe e da criança, buscando-se aqui, como condição necessária, analisar a implantação do programa, compreendendo-o como parte de um contexto em que diversos fatores para além das ações técnicas específicas, interferem no desempenho do programa. De maneira geral, quando o acesso às consultas foi efetivado, a adequação foi satisfatória, considerando-se a realização dos procedimentos previstos.

A existência de fatores limitantes ao acesso precoce à consulta médica e ao cumprimento do calendário de consultas sugere a persistência de iniqüidades que em parte podem ser equacionados por melhora no desempenho do programa, mas dependem também do desenvolvimento de um conjunto de intervenções em áreas diversas que contribuam para reduzir a iniqüidade social e de saúde em geral, e da ação continuada, pois a descontinuidade dos programas tem sido um dos grandes flagelos da nossa história 39,40. A iniqüidade social e na atenção à saúde prejudica os impactos tecnicamente possíveis (mais importantes para a saúde materna do que a saúde infantil) dos programas de atenção pré-natal em curto prazo por mecanismos extremamente complexos, recomendando grande cuidado nas análises desenvolvidas 41,42 .

\section{Resumo}

A assistência pré-natal tem sido alvo de avaliações acerca dos seus componentes essenciais e impactos em contextos diversos. Em 1999, um novo programa de atenção pré-natal para as mulheres usuárias do SUS passou a vigorar no Município de Curitiba, Paraná, Brasil, com modificações no fluxo de atendimento, número e distribuição de consultas, e procedimentos necessários a cada consulta, criando a oportunidade de realizar uma pesquisa com o objetivo de avaliar a sua implantação efetiva, tomando por referência as diretrizes técnicas estabelecidas pelo programa. O estudo abrangeu uma coorte de 660 primigestas, inscritas antes da vigésima semana de gravidez, entre março e agosto de 2000, com informações obtidas por meio de duas entrevistas domiciliares, consulta no cartão da gestante ou prontuário ambulatorial, e no prontuário hospitalar. A disponibilidade, acessibilidade e seguimento do protocolo pelos profissionais demonstramse satisfatórias, mas mantém-se um início tardio e distribuição inadequada das consultas. Na classificação de adequação geral adotada, apenas 38,6\% das mulheres preencheram todos os requisitos. Os resultados contribuem para a compreensão das complexas determinações presentes no desempenho adequado de um programa de pré-natal.

Cuidado Pré-Natal; Serviços de Saúde; Avaliação dos Serviços

\section{Colaboradores}

D. S. Carvalho e H. M. D. Novaes participaram da elaboração e redação do artigo.

\section{Referências}

1. Novaes HMD. Epidemiologia e avaliação em serviços de atenção médica: novas tendências na pesquisa. Cad Saúde Pública 1996; 12 Suppl 2:7-12.

2. Hartz ZMA. Institutionalizing the evaluation of health programs and policies in France: cuisine internationale over fast food and sur mesure over ready-made. Cad Saúde Pública 1999; 15:229-60.

3. Novaes HMD. Avaliação de programas, serviços e tecnologias em saúde. Rev Saúde Pública 2000; 34:547-9.

4. D'Oliveira AFPL, Senna DM. Saúde da mulher. In: Schraiber LB, Nemes MIB, Gonçalves RBM, organizadores. Saúde do adulto: programas e ações na unidade básica. São Paulo: Editora Hucitec; 1996. p. 86-108.

5. Novaes HMD. Puericultura em questão [Dissertação de Mestrado]. São Paulo: Faculdade de Medicina, Universidade de São Paulo; 1979.

6. Oakley A. The captured womb. Oxford: Basil Blackwell; 1984.

7. Lumbiganon P. Appropriate technology: antenatal care. Int J Gynaecol Obstet 1998; 63 Suppl 1:S91-S5.

8. Villar J, Bergsjö P. Scientific basis for the content of routine antenatal care. I. Philosophy, recent studies, and power to eliminate or alleviate adverse maternal outcomes. Acta Obstet Gynecol Scand 1997; 76:1-14.

9. Bergsjö P, Villar J. Scientific basis for the content of routine antenatal care. I. Power to eliminate or alleviate adverse newborn outcomes; some spectial conditions and examinations. Acta Obstet Gynecol Scand 1997; 76:15-25.

10. Laurenti R. Perfil da mortalidade materna. In: Minayo MCS, organizador. Os muitos Brasis: saúde e população na década de 80. Rio de Janeiro: Editora Hucitec/ABRASCO; 1995. p. 304-19. 
11. Victora CG, Barros FC. Infant mortality due to perinatal causes in Brazil: trends, regional patterns and posible interventions. São Paulo Med J 2001; 119:33-42.

12. Victora CG, Vaughan JP, Barros FC, Silva AC, Tomasi E. Explaining trends in inequities: evidence from brazilian child health studies. Lancet 2000; 356:1093-8.

13. Braveman PA. Monitoring equity in health and healthcare: a conceptual framework. J Health Popul Nutr 2003; 21:181-92.

14. Villar J, Bakketeig L, Donner A, al-Mazrou Y, B'aqeel H, Belizan JM, et al. The WHO antenatal care randomised controlled trial: rationale and study design. Paediatr Perinat Epidemiol 1998; 12 Suppl 2:27-58.

15. Carroli G, Villar J, Piaggio G, Khan-Neelofur D, Gülmezoglu M, Mugford M, et al. WHO systematic review of randomised controlled trials of routine antenatal care. Lancet 2001; 357:1565-70.

16. Alexander GR, Kotelchuck M. Quantifying the adequacy of prenatal care: a comparison of indices. Public Health Rep 1996; 111:408-19.

17. McDonagh M. Is antenatal care effective in reducing maternal morbidity and mortality? Health Policy Plan 1996; 11:1-15.

18. Misra DP, Guyer B. Benefits and limitations of prenatal care: from counting visits to measuring content. JAMA 1998; 279:1661-2.

19. Belizàn JM, Farnot U, Carroli G, al-Mazrou Y. Antenatal care in developing countries. Paediatr Perinat Epidemiol 1998; 12 Suppl 2:1-3.

20. Secretaria Municipal de Saúde de Curitiba. Valorizando a dignidade materna: diagnóstico das condições de assistência ao pré-natal, parto e puerpério, no Município de Curitiba. Curitiba: Secretaria Municipal de Saúde de Curitiba; 1996.

21. Jiménez EJB, Soares ECXF, Simão MBG, Shimazaki ME, Ducci L. Avançando na atenção maternoinfantil - Programa "Mãe Curitibana”. In: Ducci L, Simão MBG, Moisés SG, organizadores. Curitiba: a saúde de braços abertos. Rio de Janeiro: CEBES; 2001. p. 201-12.

22. McGlynn EA. Quality assessment of reproductive health services. West J Med 1995; 163:19-27.

23. Carroli G, Rooney C, Villar J. How effective is antenatal care in preventing maternal mortality and serious morbidity? An overview of the evidence. Paediatr Perinat Epidemiol 2001; 15 Suppl 1:1-42.

24. Associação Médica Brasileira. Projeto Diretrizes. Assistência pré-natal. http://www.portalmedico. org.br/diretrizes/100diretrizes/pre-natal.pdf (acessado em 20/Fev/2004).

25. Enkin MW. Randomized controlled trials in the evaluation of antenatal care. Int J Technol Assess Health Care 1992; 8 Suppl 1:40-5.

26. Munjanja SP, Lindmark G, Nyström L. Randomised controlled trial of a reduced-visits programme of antenatal care in Harare, Zimbabwe. Lancet 1996; 348:364-9.

27. Villar J, Ba'aqel H, Piaggio G, Lumbiganon P, Belizan JM, Farnot U, et al. WHO antenatal care randomised trial for the evaluation of a new model of routine antenatal care. Lancet 2001; 357:1551-64.

28. Dias-da-Costa JS, Madeira AC, Luz RM, Britto MA. Auditoria médica: programa de pré-natal em posto de saúde na região sul do Brasil. Rev Saúde Pública 2000; 34:329-36.

29. Silveira DS, Santos IS, Costa JSD. Atenção pré-natal na rede básica: uma avaliação da estrutura e do processo. Cad Saúde Pública 2001; 17:131-9.

30. Ribeiro ERRO, Barbieri MA, Bettiol H, Silva AAM. Comparação entre duas coortes de mães adolescentes em municípios do Sudeste do Brasil. Rev Saúde Pública 2000; 34:136-42.

32. Pinto-e-Silva JL. Pregnancy during adolescence: wanted vs. unwanted. Int J Gynaecol Obstet 1998; 63 Suppl 1:S151-6.

31. Gama SGN, Szwarcwald CL, Leal MC, Theme-Filha MM. Gravidez na adolescência como fator de risco para baixo peso ao nascer no Município do Rio de Janeiro, 1996 a 1998. Rev Saúde Pública 2001; 35:74-80.

33. Coimbra LC, Silva AMM, Mochel EG. Fatores associados à inadequação do uso da assistência pré-natal. Rev Saúde Pública 2003; 37:456-62.

34. Neumann NA. Cobertura, qualidade e eqüidade da atenção pré-natal e ao parto, segundo a forma de pagamento destes serviços em Criciúma-SC [Tese de Doutorado]. São Paulo: Faculdade de Saúde Pública, Universidade de São Paulo; 2000.

35. Silva AAM, Coimbra LC, Silva RA, Alves MTSSB, Lamy Filho F, Lamy ZC, et al. Perinatal health and mother-child health care in the municipality of São Luís, Maranhão State, Brazil. Cad Saúde Pública 2001; 17:1412-23.

36. Puccini RF, Pedroso GC, Silva, EMK, Araújo NS, Silva NN. Eqüidade na atenção pré-natal e ao parto em área da Região Metropolitana de São Paulo, 1996. Cad Saúde Pública 2003; 19:35-45.

37. Halpern R, Barros FC, Victora CG, Tomasi E. Atenção pré-natal em Pelotas, Rio Grande do Sul, Brasil, 1993. Cad Saúde Pública 1998; 14:487-92.

38. Trevisan MR, De Lorenzi DRS, Araújo NM, Ésber K. Perfil da assistência pré-natal entre usuárias do Sistema Único de Saúde em Caxias do Sul. Rev Bras Ginecol Obstet 2002; 24:293-9.

39. Veugelers PJ, Yip AM. Socioeconomic disparities in health care use: does universal coverage reduce inequalities in health? J Epidemiol Community Health 2003; 57:424-8.

40. Victora CG, Wagstaff A, Schellenberg JA, Gwatkin D, Claeson M, Habicht JP. Applying an equity lens to child health and mortality: more of the same is not enough. Lancet 2003; 362:233-41.

41. Dubay L, Joyce T, Kaestner R, Kenney G. Changes in prenatal care timing and low birth weight by race and socioeconomic status: implications for the medicaid expansions for pregnant women. Health Serv Res 2003; 36:373-97.

42. McCormick MC, Siegel JE. Recent evidence on the effectiveness of prenatal care. Ambul Pediatr 2001; 1:321-5.

Apresentado em 25/Mai/2004

Versão final reapresentada em 29/Jun/2004

Aprovado em 07/Jul/2004 